\title{
Correction to: Study on the Coordination Degree Between FDI and Modern Service Industry Development in Shenzhen
}

\author{
Xiangbo Zhu, Guoliang Ou, and Gang Wu
}

\begin{abstract}
Correction to:
Chapter "Study on the Coordination Degree Between FDI and Modern Service Industry Development in Shenzhen" in: Y. Xia and L.-J. Zhang (Eds.): Services - SERVICES 2019, LNCS 11517, https://doi.org/10.1007/978-3-030-23381-5_11
\end{abstract}

In the original version of the chapter titled "Study on the Coordination Degree Between FDI and Modern Service Industry Development in Shenzhen", the number of the Humanities and Social Sciences Youth Project of Hubei Provincial Department of Education was corrected from 17Q065 to 17Q056 in the acknowledgement. 